J. Clin. Chem. Clin. Biochem.

Vol. 19, 1981, pp. 31-34

\title{
Mikromethode zur Bestimmung von N-Acetyl-Neuraminsäure in Glykoproteinen
}

\author{
Von R. Kattermann und Ruth Krieger \\ Klinisch-Chemisches Institut, Klinikum Mannheim der Universität Heidelberg
}

(Eingegangen am 25. Juli 1980)

Zusammenfassung: Es wird eine Mikromethode zur Bestimmung freier bzw. gebundener N-Acetyl-Neuraminsäure (NANA) in Glykoproteinen auf der Grundlage der Periodatoxidation und Thiobarbituratreaktion beschrieben. Die Methode zeichnet sich durch einfache Durchführbarkeit bei Verwendung des Mikrolitersystems Eppendorf aus. Die Nachweisempfindlichkeit liegt bei 0,2 nmol NANA im Testansatz. Die Präzision in der Serie beträgt 3,0\%, die Langzeitpräzision 4,3\% (VK). Die Richtigkeit der erhaltenen Ergebnisse wurde durch Vergleichsuntersuchungen mit der Makromethode nach Warren ((1959), J. Biol. Chem. 234, 1971-1979) an Referenzmaterial (Sialyllactose, Transferrin, Fibrinogen) unter Beweis gestellt.

\section{A micromethod for the determination of $N$-acetylneuraminic acid in glycoproteins}

Summary: A micromethod is described for the determination of free or glycoprotein-bound $\mathrm{N}$-acetylneuraminic acid (NANA) on the basis of oxidation with periodic acid and reaction with thiobarbiturate. When using the Eppendorf microliter system the method is easy to perform, the lowest level of sensitivity being 0.2 nmoles NANA in the incubation mix ture. The intraassay precision comprises $3.0 \% \mathrm{CV}$, the interassay precision $4.3 \% \mathrm{CV}$. The accuracy of the results obtained by this micromethod was proved by comparative experiments with the macromethod according to Warren ((1959), J. Biol. Chem. 234, 1971-1979) using sialyllactose, transferrin and fibrinogen as reference materials.

\section{Einführung}

Die Biochemie der Sialinsäuren, insbesondere der N-Acetyl-Neuraminsäure (NANA) hat in der Glykoproteinforschung, aber auch in der Zellbiologie, Endokrinologie und Klinischen Chemie zunehmende Bedeutung erlangt (1-4). Im Rahmen eigener Untersuchungen zum Vorkommen von Kohlenhydratkomponenten in menschlichen und tierischen Lipoproteinen $(5,6)$ ergab sich dije Fordèrung nach einer einfachen, empfindlichen Mikromethode zur NANA-Bestimmung in LipoproteinFraktionen des Serums. Im folgenden beschreiben wir eine derartige Methode, die im Vergleich zur Makromethode nach Warren (7) um einen Faktor 5,4 empfindlicher ist und sich durch einfache Durchführbarkeit und sehr gute Zuverlässigkeitskriterien auszeichnet. In der vorliegenden Arbeit werden Untersuchungsergebnisse an reinen Glykoproteinen (Transferrin, Fibrinogen) mitgeteilt. Die Anwendung auf isolierte Lipoproteine bei gleichzeitiger Bestimmung von Hexosen und Hexosaminen ist Gegenstand einer weiteren Arbeit in dieser Zeitschrift (8).

\section{Methodik}

\section{Probenmaterial und Reagenzien}

N-Acetylneuraminsäure (NANA), 3X rekristallisiert, wurde uns freundlicherweise von Herrn Prof. Dr. Dr. $R$. Brossmer, Heidelberg, zur Verfügung gestellt. Für vergleichende NANA-Bestimmungen in biologischem Material setzten wir Sialyllactose (Boehringer Mannheim), Human-Transferrin (Sigma München) und Human-Fibrinogen (Behringwerke Marburg) ein. In einer weiteren Untersuchungsreihe wurden gefriergetrocknete Seren verschiedener Tierspezies $\mathrm{Fa}$. Nordic Immunology/Byk-Mallinckrodt, Dietzenbach), sowie gepooltes Humanserum aus dem eigenen Labor verwendet.

Die folgenden Lösungen wurden aus p.a.-Reagentien der Fa. Merck, Darmstadt hergestellt:

Lösung A: $61,0 \mathrm{ml}$ o-Phosphorsäure $(850 \mathrm{~g} / \mathrm{kg})$ mit dest. Wasser ad $100 \mathrm{ml}$ verdünnen, darin $4,28 \mathrm{~g}$ Natrium-Metaperiodat lösen $=0,2 \mathrm{~mol} / 1$ Periodat in $9 \mathrm{~mol} / 1$ Phosphosäure.

Lösung B: $7,1 \mathrm{~g}$ Natriumsulfat und $10 \mathrm{ml} 0,5 \mathrm{~mol} / 1$ Schivefelsäure mit $80 \mathrm{ml}$ dest. Wasser lösen, $20,0 \mathrm{~g}$ Natriumarsenit zugeben und ad $100 \mathrm{ml}$ auffüllen $=1,54 \mathrm{~mol} / \mathrm{l}$ Natriumarsenit und $0,5 \mathrm{~mol} / 1 \mathrm{Natriumsulfat,}$ beides gelöst in $0,05 \mathrm{~mol} / 1 \mathrm{H}_{2} \mathrm{SO}_{4}$.

Lösung C: $17,75 \mathrm{~g}$ Natriumsulfat mit etwa $240 \mathrm{ml}$ dest. Wasser lösen, $1,5 \mathrm{~g}$ Thiobarbitursäure einwiegen und ad $250 \mathrm{ml}$ auffüllen $=0,037 \mathrm{~mol} / 1$ Thiobarbitursäure in $0,5 \mathrm{~mol} / 1$ Natriumsulfat. 
Standard: $6,18 \mathrm{mg}$ NANA werden mit bidest. Wasser ad $20,0 \mathrm{ml}$ gelöst und in kleinen Portionen bei $-30^{\circ} \mathrm{C}$ eingefroren. Konzentration: $1,0 \mathrm{mmol} / \mathrm{l}$.

Die gleichen Lösungen bzw. Konzentrationen werden in der Makromethode nach Warren (7) verwendet bis auf Natriumarsenit, dessen Konzentration $0,77 \mathrm{~mol} / 1$ betragen muß.

\section{Durch führung}

\section{Mikromethode}

Der unten angegebene Testansatz läßt sich relativ einfach mit dem Mikrolitersystem 5300/5400 der Fa. Eppendorf-Gerätebau, Hamburg durchfüren. Wegen eines Gesamtvolumens von $1,57 \mathrm{ml}$ im Ansatz ist die Verwendung großer 2,2 ml-Reaktionsgefäße (Best.-Nr. 3812) von Vorteil.

Zur Hydrolyse der Glykoprotein-gebundenen NANA werden $100 \mu$ l Probe (NANA-Gehalt zwischen 0,5 und 20,0 nmol) mit $20 \mu \mathrm{l}$ einer $0,05 \mathrm{~mol} / 1 \mathrm{H}_{2} \mathrm{SO}_{4}$ versetzt, gemischt und $60 \mathrm{~min}$ bei geschlossenem Deckel in einem Heizblock oder Wasserbad bei $80^{\circ} \mathrm{C}$ inkubiert. Nach Abkühlen wird das Hydrolysat nach folgendem Arbeitsschema der NANA-Bestimmung zugeführt:

\begin{tabular}{llcl}
\hline & Leerwert & Standard & Probe \\
\hline Standardlösung & - & $10 \mu 1$ & - \\
Untersuchungsmaterial & - & - & $120 \mu 1$ \\
NaCl (9 g/l) & $120 \mu 1$ & $110 \mu 1$ & - \\
Lösung A („Periodat“) & $50 \mu 1$ & $50 \mu 1$ & $50 \mu 1$
\end{tabular}

Mischen, 20 min bei Raumtemperatur stehenlassen, sodann Zugabe von

Lösung B (,Arsenit“) $\quad 100 \mu \mathrm{l} \quad 100 \mu \mathrm{l} \quad 100 \mu \mathrm{l}$

Mischen, bis die gelbe Farbe des dabei entstehenden lods verschwunden ist. Sodann Zugabe von

Lösung C (,Thiobarbiturat") $500 \mu \mathrm{l} \quad 500 \mu \mathrm{l} \quad 500 \mu \mathrm{l}$

Mischen, $15 \mathrm{~min}$ bei $95^{\circ} \mathrm{C}$ im Heizblock inkubieren, danach abkühlen lassen auf Raumtemperatur. Zusatz von

Cyclohexanon $\quad 800 \mu \mathrm{l} \quad 800 \mu \mathrm{l} \quad 800 \mu \mathrm{l}$

Der Inhalt der Reaktionsgefäße wird bei geschlossenem Deckel gründlich geschüttelt und anschließend $2 \mathrm{~min}$ bei $10000 \mathrm{~g}$ in der Mikro-Zentrifuge zentrifugiert. Die Absorption des klaren, rötlich gefärbten Cyclohexanon-Überstandes wird in einer HalbmikroGlasküvette $(\mathrm{d}=1 \mathrm{~cm})$ bei $546 \mathrm{~nm}$ gegen den Leerwert gemessen. Bei Auftreten von Trübungen ist der Überstand in einem zweiten Reaktionsgefäß vor der Messung erneut zu zentrifugieren. Der Farbstoff bleibt etwa 3 Stunden stabil.

\section{Berechnung}

$$
\frac{\text { Aprobe }}{\text { AStandard }} \times 100=\mu \mathrm{mol} / 1 \text { NANA }
$$

\section{Makromethode}

Hier folgten wir den Angaben der Originalarbeit von Warren (7), bei der die Glykoproteinprobe mit einem NANA-Gehalt zwischen 10 und $100 \mathrm{nmol}$ in $100 \mu \mathrm{l}$ bidest. Wasser gelöst und mit $100 \mu \mathrm{l}$ einer $0,1 \mathrm{~mol} / 1 \mathrm{H}_{2} \mathrm{SO}_{4}$ versetzt wird. Die Proben werden gemischt und in einem graduierten Zentrifugenglas mit Schliffstopfen $60 \mathrm{~min}$ bei $80^{\circ} \mathrm{C}$ in einem Wasserbad hydrolysiert. Nach Abkühlen auf Raumtemperatur wird das Hydrolysat nach folgendem Arbeitsschema deṙ NANA-Bestimmung zugeführt:

\begin{tabular}{llcl}
\hline & Leerwert & Standard & Probe \\
\hline Standardlösung & - & $50 \mu 1$ & - \\
Untersuchungsmaterial & - & - & $200 \mu \mathrm{l}$ \\
NaCl $(9$ g/1) & $200 \mu 1$ & $150 \mu 1$ & - \\
Lösung A (,Periodat“) & $100 \mu \mathrm{l}$ & $100 \mu \mathrm{l}$ & $100 \mu \mathrm{l}$
\end{tabular}

Mischen, $20 \mathrm{~min}$ bei Raumtemperatur stehen lassen, sodann

Zugabe von

Lösung B („Arsenit") $\quad 1000 \mu \mathrm{l} \quad 1000 \mu \mathrm{l} \quad 1000 \mu \mathrm{l}$

Mischen, bis die gelbe Farbe des Iods verschwunden ist, södann Zugabe von

Lösung C (,Thiobarbiturat") $3,0 \mathrm{ml} \quad 3,0 \mathrm{ml} \quad 3,0 \mathrm{ml}$

Mischen, Zentrifugengläser mit Glaskugeln abdecken und $15 \mathrm{~min}$ bei $100^{\circ} \mathrm{C}$ im siedenden Wasserbad inkubieren. Nach Abkühlen auf Raumtemperatur Zugabe von

Cyclohexanon $4,3 \mathrm{ml} \quad 4,3 \mathrm{ml} \quad 4,3 \mathrm{ml}$

Die Gläser werden mit den Schliffstopfen verschlossen, manuell oder auf einer Schüttelmaschine kräftig geschüttelt und zur Phasentrennung in einer Zentrifuge $10 \mathrm{~min}$ bei $2000 \mathrm{~min}^{-1}$ zentrifugiert. Die Absorption des klaren Cyclohexanon-Überstands wird in einer normalen Glasküvette $(\mathrm{d}=1 \mathrm{~cm})$ bei $546 \mathrm{~nm}$ gegen den Leerwert gemessen.

\section{Berechnung}

$$
\frac{\text { AProbe }}{\text { Astandard }} \times 500=\mu \mathrm{mol} / 1 \text { NANA }
$$

Die Ausgangs- bzw. Endkonzentrationen der Reagentien in der wäßrigen Phase der Mikro- und der Makromethode sind in Tabelle 1 zusammengestellt.

\section{Ergebnisse und Diskussion}

Bei der hier beschriebenen Mikrobestimmung der freien bzw. gebundenen N-Acetyl-Neuraminsäure (NANA) erhält man mit der Thiobarbituratmethode nach Warren (7) einen roten Farbstoff, dessen Spektrum in Cyclohexanon mit dem der Originalmethode identisch ist. Es zeigt ein Maximum bei $\lambda=549 \mathrm{~nm}$, so daß unter Verwendung des Spektrallinien-Photometers mit Hg-Lampe sehr günstig bei $546 \mathrm{~nm}$ gemessen werden kann.

\section{Empfindlichkeit und Linearität}

Durch Erhöhung des Probenanteils im Testansatz von 1:43 auf 1:7,7 läßt sich bei nur wenig veränderten Endkonzentrationen der Reagentien die Empfindlichkeit

Tab. 1. NANA-Bestimmung mittels Makro- und Mikromethode: Ausgangskonzentration der Reagentien und Endkonzentration in der wäßrigen Phase der jeweiligen Testansätze (s. Methoden).

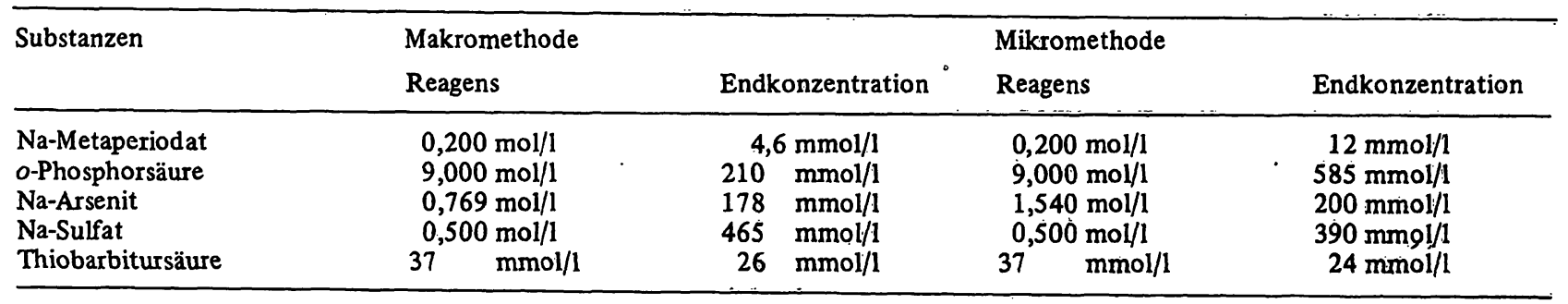


um einen Faktor 5,4 steigern (s. Abb. 1). Dies bedeutet, daß noch 0,2 nmol NANA im Testansatz mit ausreichender Genauigkeit bestimmt werden kann. Die Steigerung der Empfindlichkeit bedeutet naturgemäß, daß die Linearitätsgrenze absinkt: Oberhalb von $20 \mathrm{nmol}$ NANA im Testansatz muß die Probe verdünnt oder weniger Probe in den Test eingesetzt werden. Derart hohe NANA-Gehalte kommen aber weder in den Glykoproteinen des Serums, noch in den in der Ultrazentrifuge flotierten Lipoproteinfraktionen vor.

\section{Präzision}

Trotz mehrfacher Pipettierungen und einer photometrischen Messung in organischer Phase zeichnet sich die Mikromethode bei sorgfältiger Durchführung durch eine gute Präzision aus: Der wäßrige NANA-Standard mit 5,0 nmol im Ansatz ergab einen Variationskoeffizienten von $3,06 \%$ in der Serie $(n=10)$ und von $4,30 \%$ über einen Zeitraum von 3 Monaten $(n=21)$. Die unter Einbeziehung der Säurehydrolyse mit Sialyllactose bzw. Fibrinogen erhaltenen Präzisionen lagen in einem ähnlich guten Bereich (s. Tab. 2). Entsprechende Untersuchungen mit der Makromethode wurden nicht durchgefuihrt.

\section{Richtigkeit}

Es wurden folgende Substanzen mit der Mikrowaage eingewogen und mit physiologischer $\mathrm{NaCl}$-Lösung auf eine

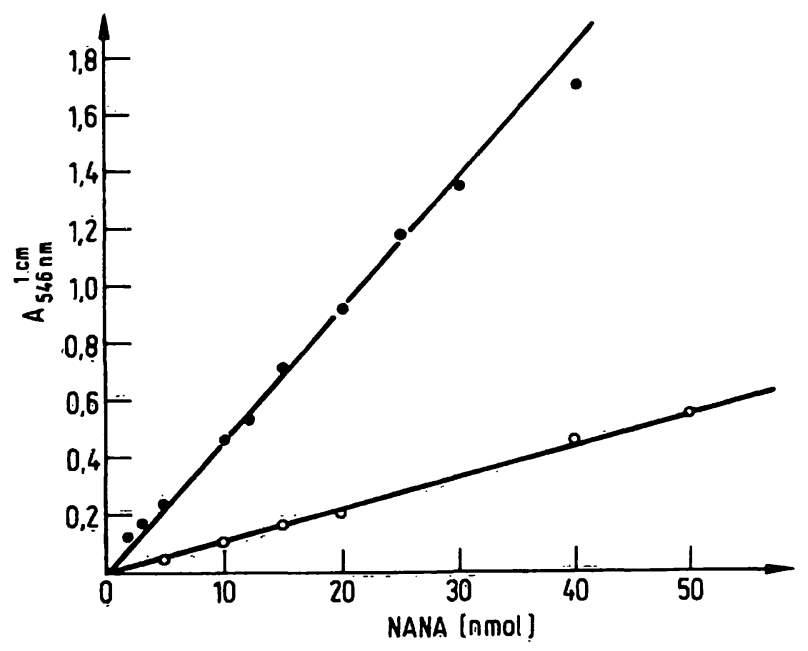

Abb: 1. Kolorimetrische Bestimmung der N-Acetyl-Neuraminsäure (NANA) mit der Thiobarbituratmethode nach Warren (7). Vergleich der Original-Makromethode $(0-0)$ mit der hier beschriebenen Mikromethode $(\bullet-\bullet)$.

Tab. 2. Präzision der NANA-Bestimmung mit der beschriebenen Mikromethode.

\begin{tabular}{lcc}
\hline $\mathrm{n}=10$ & Sialyllactose & Fibrinogen \\
\hline Mittelwert (x) & $106 \mu \mathrm{mol} / 1$ & $86,0 \mu \mathrm{mol} / 1$ \\
Standardabweichung (s) & $2,79 \mu \mathrm{mol} / 1$ & $2,51 \mu \mathrm{mol} / 1$ \\
Variationskoeffizient (VK) & $2,62 \%$ & $2,91 \%$ \\
\hline
\end{tabular}

Konzentration von 1,0 mmol/1 gelöst: Sialyllactose, Fibrinogen und Transferrin. Von diesen Lösungen wurden steigende Mengen (5-25 nmol) in die Makromethode nach Warren (7) eingesetzt. Die hierbei über einen jeweils mitgeführten Standard errechneten NANA-Gehalte wurden als Referenzwerte angesehen und mit den entsprechenden Werten der Mikromethode verglichen. Aus Abbildung 2 geht hervor, daß innerhalb der Fehlerbreite der Methoden eine sehr gute Übereinstimmung der gemessenen NANA-Gehalte besteht. Dies gilt insbesondere für die Sialyllactose und das Transferrin, während für das Fibrinogen bei der Mikromethode im gesamten Konzentrationsbereich zu niedrige NANA-Gehalte resultieren.

\section{Vergleichende Untersuchungen im Serum}

Mit der hier beschriebenen Mikromethode wurden eine Reihe gefriergetrockneter Seren auf ihren NANA-Gehalt untersucht. Die Ergebnisse sind in Tabelle 3 zusammen-

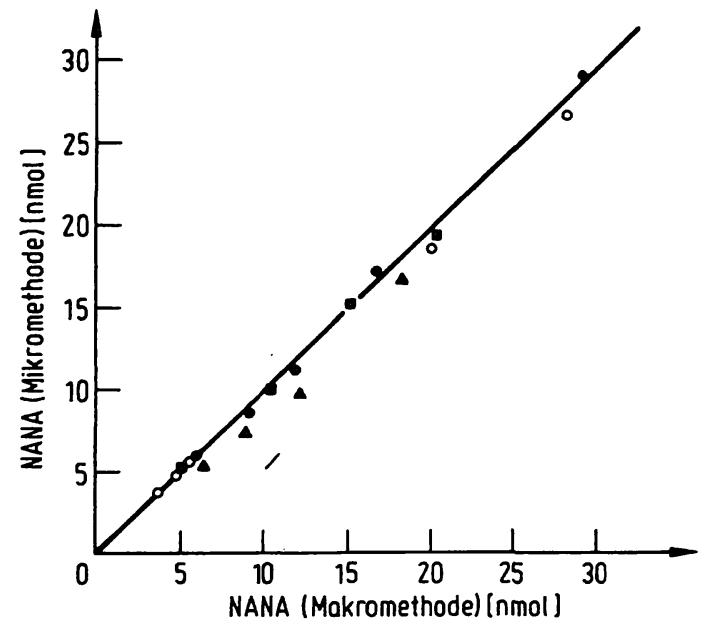

Abb. 2. Vergleichende Bestimmung des NANA-Gehaltes in wäßrigen Standards, sowie den 3 Referenzmaterialien Sialyllactose, Transferrin und Fibrinogen. Der NANA-Gehalt von Fibrinogen wird mit der Mikromethode um etwa $15 \%$ zu niedrig gemessen. Die übrigen Meßverte liegen auf oder beidseits der Identitätslinie.

- NANA-Standard Sialyl-Lactose

- Transferrin $\quad$ Fibrinogen

Tab. 3. Nach Hydrolyse gemessene NANA-Konzentrationen im Serum des Menschen und verschiedener Tierspezies unter Berücksichtigung des Totalproteingehaltes.

\begin{tabular}{llll}
\hline & NANA & $\begin{array}{l}\text { Total- } \\
\text { protein } \\
(\mathrm{g} / \mathrm{l})\end{array}$ & $\begin{array}{l}\text { NANA/Total- } \\
\text { protein } \\
\mu \mathrm{mol} / \mathrm{g}\end{array}$ \\
\hline Ente & 1,39 & 35,8 & 38,9 \\
Huhn & 1,49 & 35,6 & 41,8 \\
Taube & 1,78 & 31,9 & 49,4 \\
Rind & 2,19 & 68,8 & 31,9 \\
Schwein & 2,26 & 64,3 & 35,0 \\
Hund & 2,45 & 55,8 & 43,8 \\
Kaninchen & 2,56 & 65,1 & 39,3 \\
Pferd & 2,77 & 71,0 & 39,0 \\
Mensch & 3,09 & 78,0 & 39,6 \\
Ratte & 3,78 & 54,6 & 69,3 \\
Neugeb. Kalb & 5,18 & 53,6 & 96,7 \\
\hline
\end{tabular}


gestellt. Es zeigt sich, daß die meisten Seren eine NANAKonzentration zwischen 1,5 und 2,5 mmol/1 aufweisen. Lediglich das Serum von neugeborenen Kälbern liegt mit einer Konzentration von etwa $5,2 \mathrm{mmol} / 1$ doppelt so hoch. Die Unterschiede zwischen den einzelnen Tierspezies verwischen sich aber, wenn man die gemessene NANA auf das Totalprotein bezieht. Ratten- bzw. Kälberserum liegt aber auch hier mit einem NANA-Gehalt von rund 70 bzw. $100 \mu \mathrm{mol} / \mathrm{g}$ Protein in einem 2-3fach höheren Bereich als die übrigen Tierspezies (s. Tab. 3).

Nach unseren bisherigen Erfahrungen eignet sich die Mikromethode sowohl für die zuverlässige NANA-Bestim-

Mit Unterstützung der Deutschen Forschungsgemeinschaft (Ka 388/4).

\section{Literatur}

1. Heide, K. \& Schwick, H. G. (1973), Angew. Chemie 85, 803-815.

2. Schauer, R. (1973), Angew. Chemie 85, 128-140.

3. Ashwell, G. \& Morell, A. G. (1974), Adv. Enzymol. 41, 99128.

4. Köttgen, E., Bauer, Ch., Reutter, W. \& Gerok, W. (1979), Klin. Wochenschr. 57, 151-159, 199-214.

5. Kiss, P. \& Kattermann, R. (1979), in: Glycoconjugates, Schauer, R., Boer, P., Buddecke, E., Kramer, M. F., Vliegent- mung in säulenchromatographisch isolierten Glykoproteinen des Serums als auch für die Untersuchung des NANA-Gehaltes in den Lipoproteinfraktionen VLDL, LDL und HDL des Serums. Angesichts der von Ashwell \& Morell (3) erstmals erkannten, großen Bedeutung des Sialylierungsgrades von Glykoproteinen für deren biologische Halbwertszeit erscheint die Anwendung der NANA-Bestimmung bei Stoffwechseluntersuchungen von Lipoproteinen besonders interessant. Uber entsprechende Ergebnisse soll in einer weiteren Arbeit (8) berichtet werden. hart, I. F. G. \& Wiegandt, H. (eds.), Georg Thieme Publ, 316-317.

6. Sirowej, H., Stadler, H., Kiss, P. \& Kattermann, R. (1980), Aktuelle Endokrinologie 1, 199.

7. Warren, L. (1959), J. Biol. Chem. 234, 1971-1979.

8. Sirowej, Krieger, R. \& Kattermann, R. (1981), Manuskript in Vorberreitung.
Prof. Dr. R. Kattermann Klinisch-chemisches Institut Klinikum Mannheim Theodor-Kutzer-Ufer D-6800 Mannheim 1 